\title{
The acute effect of D-tagatose on food intake in human subjects
}

\author{
Benjamin Buemann*, Søren Toubro, Anne Raben, John Blundell and Arne Astrup \\ Research Department of Human Nutrition \& Centre for Food Research, \\ The Royal Veterinary and Agricultural University, 1958 Frederiksberg, Denmark
}

(Received 5 July 1999 - Revised 22 November 1999 - Accepted 6 January 1999)

\begin{abstract}
A double-blind randomized crossover study was performed with nineteen normal-weight men to investigate the effect on subsequent ad libitum food intake of replacing $29 \mathrm{~g}$ sucrose with $29 \mathrm{~g}$ D-tagatose as sweetener to a breakfast meal. D-Tagatose is a malabsorbed stereoisomer of fructose with potential application as a bulk sweetener. Food intake was measured at lunch offered $4 \mathrm{~h}$ after the breakfast meal, during the afternoon with access to abundant snacks, and finally at a supper buffet $9 \mathrm{~h}$ after the breakfast. Energy intake at lunch and during the snacking period was similar after ingesting the two sugars, while it was $15 \%$ lower after ingesting D-tagatose than with sucrose at supper $(P<0.05)$. Gastrointestinal factors such as the osmotic effects of unabsorbed D-tagatose causing distension of the gut might have mediated the acute appetite-suppressing effect. The present paper also refers to data from a preceding study in which we observed an increased self-reported energy intake after ingestion of D-tagatose compared with sucrose which, in fact, suggests a relative hyperphagic effect of D-tagatose. However, self-reported food intake may be biased by selective under-reporting and this subsequent study with a more controlled assessment of food intake was therefore conducted. This present study did not support any hyperphagic effect of D-tagatose, but rather suggests that D-tagatose may contribute to a reduced energy intake.
\end{abstract}

Appetite: D-Tagatose: Food intake

Artificial sweeteners have been incorporated into diets for overweight human subjects to reduce their body weight. Some (Rolls, 1991), but not all (Lavin et al. 1997), studies have shown that the replacement of natural sugars with intense sweeteners such as aspartame can reduce total ad libitum energy intake. Furthermore, aspartame has been shown to introduce a minor weight loss when replacing sucrose during ad libitum energy intake (Raben et al. 1996), and to facilitate long-term weight maintenance after weight loss (Blackburn et al. 1997).

As an alternative to intense sweeteners, the so-called bulk sweeteners have been introduced onto the market. They consist of modified monomers or dimers of common sugars such as sugar alcohols, or stereoisomers of fructose, and they have a sweetening effect similar to or lower than that of sucrose. They possess a reduced net energy content compared with natural sugars due to a poor digestibility and/or absorption as their special structure makes them resistant to intestinal enzymes or carrier mechanisms. However, these compounds may to some extent be absorbed and affect appetite by their metabolism. In this context, the degradation of particular sugars in the liver may be most important. Thus, the apparently greater satiating power of fructose compared with glucose has been speculated to be associated with the fact that most of the fructose, in contrast to glucose, is metabolized in the liver (Moyer \& Rodin, 1993). Generally, hepatic metabolism of different metabolites seems strongly to affect appetite (Scharrer \& Langhans, 1988).

Due to their unusual structure, the rate of each step in the degrading process may differ from that of their corresponding common isomer and this may cause metabolic perturbations in the liver which may modify hunger signals. Another factor which may affect hunger after ingestion of rare sugars is the short-chain fatty acids produced by their fermentation if they are malabsorbed. We therefore found it relevant to study the impact on food intake of a rare ketohexose in human subjects. The present study investigates the effect of D-tagatose, a malabsorbed stereoisomer of D-fructose, on subsequent ad libitum food intake. The study was performed subsequent to another study which showed an increase in self-reported energy intake when $29 \mathrm{~g}$ sucrose was substituted with $29 \mathrm{~g}$ D-tagatose (unpublished results). However, food intake data in that study were based on self-reporting which may be inaccurate due to recording errors by the subjects. We therefore found it crucial to perform a study where the appetite effect of D-tagatose was studied by the more controlled buffet approach. 


\section{Methods}

Twenty male medical students were recruited, of which one subject was excluded due to headache on the second test day. Body weight, BMI and age of the remaining nineteen subjects were 78.2 (SEM 1.8) kg, 24.0 (SEM 0.5) kg/m ${ }^{2}$ and 25.7 (SEM 0.9) years respectively. Only subjects who were able to tolerate $30 \mathrm{~g}$ D-tagatose orally without noteworthy gastrointestinal symptoms in a preceding screening test were included.

The dose of D-tagatose ( $29 \mathrm{~g}$ ) or sucrose ( $29 \mathrm{~g}$ ) was added to a $1.6 \mathrm{MJ}$ conventional continental breakfast consisting of yoghurt with cereals, buns with butter, jam, cheese, and orange juice. The jam served with the yoghurt and bread contained the test sugar. The two tests were conducted in a double-blind, balanced and randomized design separated by $4 \mathrm{~d}$ or more. The breakfast was served after a $12 \mathrm{~h}$ fast and was consumed within $20 \mathrm{~min}$. Subsequently, the subjects remained in the dining room for $4 \mathrm{~h}$ where they were supervised while they were reading study textbooks, but they were allowed to use the rest room. No food or water consumption was allowed during that period. After the $4 \mathrm{~h}$, a lunch was presented consisting of noodles and minced meat which had been thoroughly mixed before it was served. The subjects were instructed to eat ad libitum until they felt comfortable and not to be concerned about leftovers. The subjects filled their plates from a large saucepan with an abundant quantity of the homogeneously prepared meal. The subjects could refill their plates as many times as they wished. The plates were weighed before and after each filling, and when lunch was terminated. Water, which was the only beverage allowed with lunch, was supplied ad libitum, and the intake was weighed.

After lunch, the subjects were free to leave the department and resume their habitual activities, or they could remain in the lunch room. To provide constant access to abundant food between lunch and supper, the subjects were supplied with an insulated cool-box containing different snack items (Table 1). They were instructed to eat as much as they pleased of the items in the box at any time, and to put

Table 1. Contents of snack box available between lunch and supper

\begin{tabular}{ll}
\hline Item & Number and weight \\
\hline Orange juice & \\
Apple juice & $2 \times 200 \mathrm{~g}$ \\
Apricot jam & $2 \times 200 \mathrm{~g}$ \\
Raspberry jam & $2 \times 20 \mathrm{~g}$ \\
Chocolate bars & $2 \times 20 \mathrm{~g}$ \\
Biscuit with filling & $5 \times 19 \mathrm{~g}$ \\
Cream (10\% fat) & $7 \times 13.6 \mathrm{~g}$ \\
Sugar (lump) & $4 \times 7.5 \mathrm{~g}$ \\
Chocolate milk & $4 \times 6 \mathrm{~g}$ \\
Cream cheese with shrimp & $2 \times 200 \mathrm{~g}$ \\
Firm cheese & $2 \times 20 \mathrm{~g}$ \\
Butter & $2 \times 20 \mathrm{~g}$ \\
Yoghurt with fruit jam & $4 \times 10 \mathrm{~g}$ \\
Apples & $2 \times 150 \mathrm{~g}$ \\
Bananas & 2 \\
Buns & 2 \\
Sliced turkey meat & $4 \times 76 \mathrm{~g}$ \\
Sliced ham & $1 \times 30 \mathrm{~g}$ \\
\hline
\end{tabular}

leftovers and wrappings back into the box for re-weighing. Apart from the contents of the snack box, the subjects were allowed to drink tap water, but were instructed to measure its volume using a measuring-cup which was placed in the box. The subjects returned to the department for supper $5 \mathrm{~h}$ after lunch. The supper consisted of a buffet which was abundant with rissoles, hot potatoes, hot peas, white bread, hot tomato sauce, hot parsley sauce, butter, sandwiches with egg salad, sandwiches with smoked ham, cucumber, apple pie, ice cream, tap water, carbonated water, yellow and red fruit syrups. The subjects were instructed to eat as much as they pleased from all the items until they felt comfortable. No time restrictions for lunch and supper were imposed on the subjects. All subjects consumed the meals in the same room, but the commencement of the tests was separated by at least 20 min between the different subjects to minimize inter-subject interactions at the supper buffet. The first breakfast meal commenced at 08.00 and the last at 09.00 hours, with a maximum of five subjects each day. Each subject had his meals at the same time in the two different tests. During all the meals, the subjects were separated by more than $4 \mathrm{~m}$, and were not allowed to communicate with each other. Intakes of energy and of macronutrients for each day were calculated by DANKOST version 2.0 dietary assessment software (Danish Catering Centre Ltd, Søborg, Denmark). However, as the recipes used for rissole preparation may vary substantially, energy content of this component was determined by bomb calorimetry.

Gastrointestinal symptoms (heartburn, distention, nausea, vomiting, stomach-ache, rumbling in the gut, flatulence and diarrhoea) during the test days were reported on questionnaires by rating symptom severity on a five-level scale.

The study was approved by the Municipal Ethical Committee of Copenhagen and Frederiksberg, Denmark.

\section{Statistics}

Data are presented as means with standard errors of the means. Differences in intake were analysed by paired $t$ test. Statistical analyses were performed with STATGRAPHICS version 4.2 (Graphic Software Systems, Rockville, MD, USA). $P$ values $<0.05$ were regarded as significant.

\section{Results}

Energy intake was similar in the two tests at lunch and during the snack period, but lower at supper after D-tagatose (Table 2). Total energy intake after breakfast did not differ between sucrose and D-tagatose suggesting that no later compensation occurred for the putative reduction in net energy content introduced by the lower metabolizable energy of D-tagatose compared with sucrose (Table 2). Thus, by assuming that net energy of D-tagatose is $50 \%$ of that of sucrose the energy deficit of $242 \mathrm{~kJ}$ was compensated for by -490 (SEM 467 ) $\mathrm{kJ}$ or $-202 \%$ by the subsequent meals. However, the total difference in calculated net energy intake of $732($ SEM 467$) \mathrm{kJ}(=242 \mathrm{~kJ}+490 \pm 467 \mathrm{~kJ})$ did not reach statistical significance $(P$ 0.07). The lower energy intake at supper after D-tagatose could not be particularly attributed to differences in the consumption of the major dishes or desserts (Table 3). 
Table 2. Energy intake between 4 and $9.5 \mathrm{~h}$ following intake of $29 \mathrm{~g}$ sucrose or D-tagatose given as sweetener to a breakfast meal ${ }^{\star}$

(Mean values with standard errors of the means for nineteen male subjects)

\begin{tabular}{|c|c|c|c|c|c|c|}
\hline \multirow[b]{2}{*}{ Meal } & \multicolumn{2}{|c|}{ Sucrose } & \multicolumn{2}{|c|}{ D-Tagatose } & \multirow{2}{*}{$\begin{array}{l}\text { Difference in } \\
\text { energy intake } \\
\%\end{array}$} & \multirow{2}{*}{$\begin{array}{c}\text { Statistical significance } \\
\text { of difference between } \\
\text { means; } P \dagger\end{array}$} \\
\hline & Mean & SEM & Mean & SEM & & \\
\hline Lunch & 4196 & 173 & 4401 & 219 & +4.9 & 0.39 \\
\hline Snack & 5445 & 555 & 5243 & 436 & -3.7 & 0.63 \\
\hline Supper & 3411 & 264 & 2918 & 291 & -14.5 & 0.04 \\
\hline Lunch + snack + supper & 13053 & 605 & 12563 & 569 & -3.8 & 0.31 \\
\hline
\end{tabular}

* For details of subjects and meals see p. 228.

$\dagger P$ values obtained by two-sided Student's $t$ test for paired observations.

Macronutrient composition of total post-lunch food intake was unaffected by the nature of the experimental sugar (Table 4). However, when the post-lunch food intake was subdivided into snack and supper, fat intake as a percentage of energy intake at supper was lower after Dtagatose (Table 4). The sum of lunch and snack liquid consumption was $11 \%$ higher after D-tagatose $(P<0 \cdot 05)$. However, this was partly compensated for by a lower liquid intake with the supper (Table 5). The difference in fat intake as a percentage of energy intake combined with the

Table 3. Intake (g) of major dishes at supper following intake of $29 \mathrm{~g}$ sucrose or D-tagatose given as sweetener to a breakfast meal ${ }^{*}$ (Mean values with standard errors of the mean for nineteen male subjects)

\begin{tabular}{|c|c|c|c|c|c|}
\hline & \multicolumn{2}{|c|}{ Sucrose } & \multicolumn{2}{|c|}{ D-Tagatose } & \multirow{2}{*}{$\begin{array}{c}\text { Statistical significance } \\
\text { of difference between } \\
\text { means: } P \dagger\end{array}$} \\
\hline & Mean & SEM & Mean & SEM & \\
\hline Rissoles & 138 & 18 & 118 & 12 & 0.19 \\
\hline Apple pie & 18 & 6 & 13 & 5 & $0 \cdot 16$ \\
\hline Ice cream & 47 & 12 & 41 & 11 & 0.67 \\
\hline
\end{tabular}

${ }^{*}$ For details of subjects and meals, see p. 228.

$\dagger P$ values obtained by two-sided Student's $t$ test for paired observations.

Table 4. Macronutrient composition (percentage of energy) consumed as snack and at supper following intake of $29 \mathrm{~g}$ sucrose or $D$-tagatose given as sweetener to a breakfast meal $\dagger$

(Mean values with standard errors of the mean for nineteen male subjects)

\begin{tabular}{|c|c|c|c|c|}
\hline & \multicolumn{2}{|c|}{ Sucrose } & \multicolumn{2}{|c|}{ D-Tagatose } \\
\hline & Mean & SEM & Mean & SEN \\
\hline \multicolumn{5}{|l|}{ Snack } \\
\hline Carbohydrate & $66 \cdot 2$ & $1 \cdot 2$ & $66 \cdot 3$ & 1.4 \\
\hline Fat & $26 \cdot 2$ & $1 \cdot 2$ & $25 \cdot 4$ & 1.4 \\
\hline Protein & 7.4 & 0.6 & 8.2 & 0.4 \\
\hline \multicolumn{5}{|l|}{ Supper } \\
\hline Carbohydrate & $49 \cdot 8$ & $1 \cdot 8$ & $52 \cdot 3$ & 1.7 \\
\hline Fat & $32 \cdot 9$ & $1 \cdot 3$ & $29 \cdot 9^{*}$ & 1.3 \\
\hline Protein & $16 \cdot 7$ & 0.9 & $17 \cdot 3$ & 0.9 \\
\hline \multicolumn{5}{|l|}{ Snack + supper } \\
\hline Carbohydrate & $60 \cdot 0$ & $1 \cdot 1$ & $60 \cdot 7$ & 1.0 \\
\hline Fat & $28 \cdot 6$ & 0.9 & $27 \cdot 8$ & 0.9 \\
\hline Protein & $11 \cdot 2$ & 0.7 & $11 \cdot 3$ & 0.4 \\
\hline
\end{tabular}

Mean value was significantly different from sucrose group (two-sided Student's $t$ test for paired observations), ${ }^{*} P<0.05$.

† For details of subjects and meals see $p \cdot 228$. differences in liquid intake resulted in similar energy densities of the total supper intake (D-tagatose $v$. sucrose: $3.85($ SEM 0.20) v. $3.90($ SEM 0.21$) \mathrm{kJ} / \mathrm{g})$.

No major gastrointestinal symptoms were reported except for two cases of moderate nausea and one case of strong flatulence after D-tagatose.

\section{Discussion}

A lower intake at supper $(-15 \%)$ was found when $29 \mathrm{~g}$ sucrose was replaced by the same amount of D-tagatose as sweetener to a breakfast. This apparent appetite suppressing effect of the sugar may be attributable to gastrointestinal factors. Absorption of D-tagatose is poor (Lærke \& Jensen, 1999) and this may result in intestinal distention due to accumulation of fluid caused by the increased osmotic pressure of the intestinal juice. That D-tagatose causes water retention is supported by the previous findings of a higher plasma albumin concentration after intake of a $30 \mathrm{~g}$ D-tagatose solution relative to D-fructose or plain water (Buemann et al. 2000), indicating a haemoconcentrating effect. Haemoconcentration, in turn, increases water intake by stimulating thirst, as demonstrated in the present study by the greater liquid intake for the lunch + snack period. The increased liquid intake may have contributed to satiety by increasing gastric distention. An exaggerated response after D-tagatose on the intestinal hormones like glucose-dependent insulinotropic polypeptide, glucagon-like peptide-1 or cholecystokinin seem less likely to explain the appetite suppressing effect of the

Table 5. Water intake (g) between 4 and $9.5 \mathrm{~h}$ following intake of $29 \mathrm{~g}$ sucrose or D-tagatose given as sweetener to a breakfast meal ${ }^{*}$

(Mean values with standard errors of the mean for nineteen male subjects)

\begin{tabular}{|c|c|c|c|c|c|}
\hline & \multicolumn{2}{|c|}{ Sucrose } & \multicolumn{2}{|c|}{ D-Tagatose } & \multirow{2}{*}{$\begin{array}{c}\text { Statistical } \\
\text { significance of } \\
\text { difference between } \\
\text { means: } P+\end{array}$} \\
\hline & Mean & SEM & Mean & SEM & \\
\hline Lunch & 391 & 44 & 437 & 38 & 0.12 \\
\hline Snack period & 731 & 57 & 807 & 64 & 0.13 \\
\hline Lunch + snack & 1122 & 86 & 1245 & 80 & 0.03 \\
\hline Supper & 343 & 32 & 290 & 31 & 0.06 \\
\hline Total & 1465 & 100 & 1534 & 95 & 0.17 \\
\hline
\end{tabular}

${ }^{*}$ For details of subjects and meals, see p. 228.

$\dagger P$ values obtained by two-sided Student's $t$ test for paired observations. 
sugar, as the previous study using a similar dose of Dtagatose showed an attenuated response compared with fructose in these hormones until $7 \mathrm{~h}$ post-dose (Buemann et al. 2000). The unlimited access to palatable food in the present study may have introduced overconsumption. Total energy intake including breakfast in the sucrose trial was $31 \%$ higher $(P<0.0005)$ than $1.55 \times$ resting energy expenditure which has been suggested as the energy requirement for a sedentary lifestyle (Black et al. 1991). Resting energy expenditure was individually calculated from body weight, height and age of the subjects (Mifflin et al. 1990). Most of the subjects were sedentary during the experimental day as the majority remained in the dining room. The fact that snack intake during the afternoon alone exceeded 5 MJ suggests a passive overconsumption, promoted by the free access to highly palatable snacks. Thus, the lower intake at supper after D-tagatose was demonstrated after a sedentary $5 \mathrm{~h}$ period with a very high consumption of snacks rich in carbohydrates, which may have suppressed hunger both after sucrose and D-tagatose. The observed trend of a reduced energy intake after D-tagatose contrasts with the finding of a $30 \%$ higher $(P=0.006)$ post-lunch energy intake after D-tagatose in the self-reporting study, which was performed with thirty-three subjects and had a protocol identical to the present study, except that assessment of food intake after lunch was based on weighed dietary records (Buemann et al. 2000). The validity of the result of the selfreporting study may be questioned, as self-reported food intake may be inaccurate due to under-reporting also in normal-weight subjects (Livingstone et al. 1990). However, if the higher energy content of the self-reported intake after D-tagatose should be explained by inaccurate recording the error must be biased with respect to treatment. One possible mechanism may be that the subjects are feeling more satiated after D-tagatose, and therefore less inclined to disregard part of their consumption. However, a greater satiation cannot be confirmed as appetite variables were not measured after the subjects left the department.

The effect of D-tagatose on self-reported food intake may depend on the contemporary intake of other foods as no increase in self-reported food intake was observed in a previous study after D-tagatose compared with fructose or plain water when it was administered to fasting subjects dissolved in water as the only nutrient (Buemann et al. 2000). The observation of increased food intake by Dtagatose would be in agreement with findings that 2,5anhydro-D-mannitol, another fructose derivate, increases food intake in rats (Rawson et al. 1994). 2,5-anhydro-Dmannitol has a phosphate-trapping capacity (Riquelme et al. 1984) like D-tagatose (Vincent et al. 1986). The hyperphagic effect of 2,5-anhydro-D-mannitol has been found to be accompanied by a $37 \%$ reduction in the ATP level in the liver (Rawson \& Friedman, 1994) and it has been suggested that a declining ATP level in the liver is an important hunger signal (Friedman, 1997). By using ${ }^{31} \mathrm{P}$ magnetic resonance spectroscopy we have recently demonstrated an approximately $12 \%$ reduction in hepatic ATP in human subjects after $30 \mathrm{~g}$ D-tagatose was given orally as a watery solution (B Buemann, H Gesmar, A Astrup and B Quistorff, unpublished results) but the ATP level was back to normal after $3 \mathrm{~h}$. The increased intake after D-tagatose in the self-reporting study (B Buemann, S Toubro, A Raben and A Astrup, unpublished results) was not observed at the lunch given $4 \mathrm{~h}$ after the load but only in the post-lunch periods where it also persisted during the subsequent day. In contrast, in Rawsons \& Friedman's (1994) study the eating response to 2,5-anhydro-D-mannitol was elicited contemporary with the decline in hepatic ATP. For this reason, the presence of a hyperphagic effect directly elicited by a D-tagatose-induced ATP depletion does not seem likely to explain the greater food intake unless the absorption of D-tagatose was delayed or increased by the breakfast meal given with the load. However, such a profound effect of the modest breakfast meal on the absorption of D-tagatose does not seem plausible. However, a hyperphagic effect might be a result of later perturbations eventually secondary to an ATP depletion. In any case, the hyperphagic effect of phosphate trapping keto-hexoses may be counterbalanced with oral administration by gastrointestinal factors or may vanish with adaptation since a reduced, rather than increased food consumption, has been reported in a rat study where $20 \%$ of the chow was replaced by D-tagatose during a $90 \mathrm{~d}$ period (Kruger et al. 1999). Furthermore, no increase in body weight or fat mass was observed in a study on human subjects where $30 \mathrm{~g}$ D-tagatose was consumed during $14 \mathrm{~d}$ (Buemann et al. 1998). Fructose, with a similar in structure to D-tagatose, is also reported to have a suppressant effect on subsequent food intake, although the research is not conclusive (Moyer \& Rodin, 1993). The proposed underlying mechanisms include delayed gastric emptying, concentrations of plasma glucose and insulin and hepatic metabolism. The mechanisms for the appetite suppressant effect of D-tagatose may be different from that for fructose as only about $25 \%$ is absorbed in the small intestine (Lærke \& Jensen 1999).

\section{Conclusion}

The different outcome between the self-reporting study (unpublished results) and the present study with controlled food intake measurements may reflect the sensitivity of appetite studies. Underlying experimental factors such as how food consumption data are collected and general food availability might be very important to determine the final outcome when the effect on energy intake of a particular substance is tested. The effect of regular D-tagatose consumption in 'real-life' is therefore still unclear. Moreover, further research is needed to elucidate mechanisms behind possible hypo- or hyperphagic effects of D-tagatose.

\section{Acknowledgements}

We thank the staff of the metabolic unit of Research Department of Human Nutrition: Charlotte Kostecki, Hanne Jensen and Dorte Hansen. Special thanks to biochemical student Sarah Fredriksen and to our student in human nutrition Pernille Nielsen who assisted with the food recording and dietary calculations. We are also indebted to Hans Bertelsen, MD Foods Ingredients, Denmark and to Lee Zehner and Jim Saunders, Biospherics, USA, who assisted with the preparation of the experimental protocol and the manuscript. The work was supported by the 
Danish Research and Development Programme for Food Technology and by MD Foods Ingredients amba.

\section{References}

Black AE, Goldberg SA, Jebb SA, Livingstone MBE, Cole TJ \& Prentice AM (1991) Critical evaluation of energy intake data using fundamental principles of energy physiology: 2. Evaluating the results of published surveys. European Journal of Clinical Nutrition 45, 583-599.

Blackburn GL, Kanders BS, Lavin PT, Keller SD \& Whatley J (1997) The effect of aspartame as part of a multidisciplinary weight-control program on short- and long-term control of body weight. American Journal of Clinical Nutrition 65, 409418.

Buemann B, Toubro S \& Astrup A (1998) D-Tagatose, a stereoisomer of D-fructose, increases hydrogen production in humans without affecting 24-hour energy expenditure or respiratory exchange ratio. Journal of Nutrition 128, 1481-1486.

Buemann B, Toubro S, Holst JJ, Rehfeld JF \& Astrup A (2000) D-Tagatose, a stereoisomer of D-fructose increases blood uric acid concentration. Metabolism (In the Press).

Friedman MI (1997) An energy sensor for control of energy intake. Proceedings of the Nutrition Society 56, 41-50.

Kruger CL, Whittaker MH, Frankos VH \& Trimmer GW (1999) 90-Day oral toxicity study of D-tagatose in rats. Regulatory Toxicology and Pharmacology 29, S1-S10.

Lærke HN \& Jensen BB (1999) D-Tagatose has low small intestinal digestibility but high large intestinal fermentability in pigs. Journal of Nutrition 129, 1002-1009.

Lavin JH, French SJ \& Read NW (1997) The effect of sucrose- and aspartame- sweetened drinks on energy intake, hunger and food choice of female, moderately restrained eaters. International Journal of Obesity 21, 37-42.

Livingstone MBE, Prentice AM, Strain JJ, Coward WA, Black AE,
Barker ME, McKenna PG \& Whitehead RG (1990) Accuracy of weighed dietary records in studies of diet and health. British Medical Journal 300, 708-712.

Mifflin MD, St Jeor ST, Hill LA, Scott BJ, Daugherty SA \& Koh YO (1990) A new predictive equation for resting energy expenditure in healthy individuals. American Journal of Clinical Nutrition 51, 241-247.

Moyer AE \& Rodin J (1993) Fructose and behavior: does fructose influence food intake and macronutrient selection? American Journal of Clinical Nutrition 58, Suppl., 810S-814S.

Raben A, Tatjana HV, Møller C \& Astrup A (1996) Sucrose vs artificial sweeteners: Minor differences in body weight after 10 weeks. International Journal of Obesity 20, Suppl. 4, 51 Abstr.

Rawson NE \& Friedman MI (1994) Phosphate loading prevents the decrease in ATP and increase in food intake produced by 2,5-anhydro-D-mannitol. American Journal of Physiology 266, R1792-R1796.

Rawson NE, Blum H, Osbakken MD \& Friedman MI (1994) Hepatic phosphate trapping, decreased ATP, and increased feeding after 2,5-anhydro-D-mannitol. American Journal of Physiology 266, R112-R117.

Riquelme P, Wernette-Hammond ME, Kneer NM \& Lardy HA (1984) Mechanism of action of 2,5-anhydro-D-mannitol in hepatocytes. Effects of phosphorylated metabolites on enzymes of carbohydrate metabolism. Journal of Biological Chemistry 259, 5115-5123.

Rolls BJ (1991) Effects of intense sweeteners on hunger, food intake, and body weight: a review. American Journal of Clinical Nutrition 53, 872-878.

Scharrer E \& Langhans W (1988) Metabolic and hormonal factors controlling food intake. International Journal of Vitamin and Nutrition Research 58, 249-261.

Vincent MF, Van den Berghe G \& Hers H-G (1986) Effect of fructose on the concentration of phosphoribosylpyrophosphate in isolated hepatocytes. Advanced Experimental Medical Biology 195, Pt. B, 615-621. 